\title{
Tumor Necrosis Factor Suppression
}

National Cancer Institute

\section{Source}

National Cancer Institute. Tumor Necrosis Factor Suppression. NCI Thesaurus. Code C40601.

Any therapeutic or preventive procedure that involves inhibition of the production or activity of a Tumor Necrosis Factor. This process may result also in inhibition of some functions of mature immune cells, including polymorphonuclear chemotaxis and monocyte phagocytosis. Tumor Necrosis Factor suppression is intended to favorably modulate functions of the immune system. 